\title{
Littre's hernia- a rare case of a strangulated Meckel's diverticulum
}

\author{
Stephen-John Martin, Everett Nana Kwame Asante \\ Stephen-John Martin, Junior Medical Officer, Thelle Mogoerane Regional Hospital (Natalspruit) \\ WITS University
}

Corresponding author: Stephen-John Martin (peacefuldoctor@gmail.com)

Summary: Littre's hernia a reminder of the unusual contents of an inguinal hernia and the principles of management.

S Afr J Surg 2018;56(4)

http://dx.doi.org/10.17159/2078-5151/2018/v56n4a2864

\section{Case Report}

A 4-year-old previously healthy male child, with no significant medical or family history, presented to our on-call surgical unit with a 1-week history of a fixed left-sided groin mass and associated dysuria. On examination the child appeared well nourished and was not dehydrated. His temperature was $38.0^{\circ} \mathrm{C}$ and his heart rate 143 beats per minute. His respiratory rate and blood pressure were normal for his age. Abdominal examination was unremarkable apart from a $3 \mathrm{~cm}$ round, tender left-sided inguinal mass that was fixed but had no associated skin changes. A strangulated inguinal hernia was suspected clinically and the child was taken to the operating room for exploration of the left groin. The mass was approached with an oblique incision along the course of the inguinal ligament. The hernia sac was identified and separated from the spermatic cord, viable small bowel was discovered within the sac but small amounts of pus were noted to be emanating through the deep ring from within the abdomen and the sac was difficult to reduce. The procedure was converted to a lower midline laparotomy. An abnormal segment of small bowel roughly $55 \mathrm{~cm}$ proximal from the ileocaecal valve was found in the hernia sac and its tip which had coiled up was necrotic (Figure 1). This segment of bowel was resected and a primary anastamosis of the small bowel was performed. A herniotomy was then performed and the abdomen closed. The child was then transferred to the ward, had an uncomplicated postoperative course and was discharged after 3 days. At routine follow-up, the child was well and without any complications. Histology confirmed a Meckel's diverticulum with no heterotropic tissue present.

\section{Discussion}

The child had a Littre's hernia. The novelty of which lies in the occurrence of two distinct surgical pathologies that comprise the condition: an inguinal hernia and a strangulated Meckel's diverticulum.

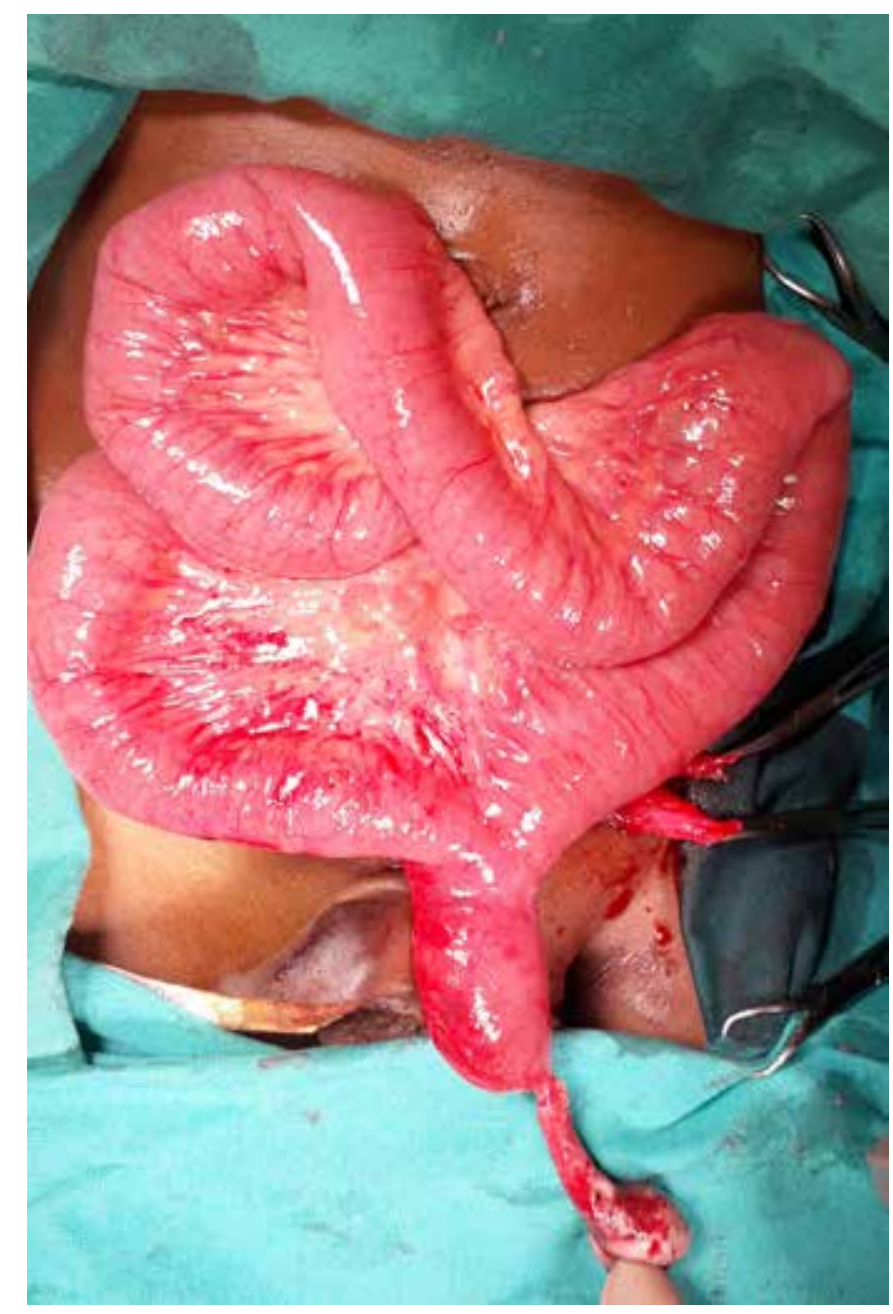

Figure 1

In the fourth week of human development a tubular vitelline or omphalomesenteric duct connects the lumen of the midgut to the yolk sac. At a stage, the communication should obliterate allowing the intestine to freely reside within the 
peritoneal cavity. Persistence of the vitelline duct, however, results in various abnormalities depending on the portion of the duct that has remained patent. If the intestinal end persists a Meckel's diverticulum is formed.

These true diverticula (outpouchings involving all layers of the gastrointestinal tract), initially reported by Hildanus in 1598 and later by Johann Meckel in 1809, have been described as following a "Rule of $2 \mathrm{~s}$ ": that is, they are found in approximately $2 \%$ of the population, are 2 inches ( 5 centimetres) in length, are found 2 feet (60 centimetres) from the ileocaecal valve and contain 2 types of ectopic mucosa gastric (in $50 \%$ of cases) and pancreatic (in 5\% of cases). Those containing gastric mucosa may result in gastrointestinal bleeding caused by peptic ulceration of adjacent portions of intestine exposed to the hydrochloric acid produced within the diverticulum. While most are found incidentally at laparotomy, barium studies or autopsy, Meckel's diverticula are also known to be complicated by inflammation (diverticulitis), perforation, obstruction (in the form of intussusception or volvulus) and rarely by incarceration or strangulation within the site of a hernia.

In 1700, the French surgeon Alexis Littre was the first to report cases of incarcerated femoral hernias containing a then un-named small bowel diverticulum. Since then Littre's hernia has been described as occurring at the inguinal, umbilical, and femoral sites. ${ }^{2}$ While this incarceration of a Meckel's diverticulum is well described, it is exceedingly rare with less than 50 cases reported in the 300 years ensuing the initial description. ${ }^{3}$ The management of Littre's hernia consists of resection of the diverticulum, anastomosis of bowel, followed by herniorrhaphy (or herniotomy in children). Care must be taken to ensure that there is minimal contamination of the hernial field, ${ }^{2}$ if good outcomes such as those experienced by the patient in the case report are to be expected.

\section{REFERENCES:}

1. Townsend C, Beauchamp R, Evers B, et al. Sabiston Textbook of Surgery: The Biological Basis of Modern Surgical Practice. 19th Ed. Philadelphia: Elsevier Saunders; 2012.

2. Skandalakis P, Zoras O, Skandalakis J, et al. Littre Hernia: Surgical Anatomy, Embryology, and Technique of Repair. Am Surg. 2006;72(3):238-43.

3. Malling B, Karlsen A, Hern J. Littre Hernia: A Rare Case of an Incarcerated Meckel's Diverticulum. Ultrasound Int Open. 2017;3:E91-E92. 